\title{
The Influence of Flowing Water on the Resource Pursuit-Risk Avoidance Tradeoff in the Crayfish Orconectes virilis
}

\author{
Keith W. Pecor*` \& Brian A. Hazlett* \\ * Department of Ecology and Evolutionary Biology, University of Michigan, Ann Arbor, MI, USA \\ $\dagger$ Museum of Zoology, University of Michigan, Ann Arbor, MI, USA
}

\author{
Correspondence \\ Biology Department, Rhodes College, 2000 \\ North Parkway, Memphis, TN 38112, USA. \\ E-mail: kpecor@umich.edu; \\ pecork@rhodes.edu
}

Received: February 15, 2005

Initial acceptance: April 8, 2005

Final acceptance: June 18, 2005

(S. Forbes)

doi: 10.1111/j.1439-0310.2005.01171.x

\begin{abstract}
The influence of hydrodynamics on chemically mediated behavioral tradeoffs has received little attention. We tested the hypothesis that individuals of the crayfish Orconectes virilis would be more sensitive to chemical cues in flowing water than in still water. Orconectes virilis is a good subject for this test, because it is found in both still water (e.g. ponds), and flowing water (e.g. rivers). A factorial design was used, with two stimulus treatments and two habitat types. Crayfish were exposed to either food cue or food + alarm cue in either still water or flowing water in an artificial stream arena. Habitat use and activity were significantly influenced by stimulus treatment, with more time spent away from the stimulus source and less activity in the food + alarm treatment than in the food treatment. Neither habitat type nor the interaction of stimulus treatment and habitat type had a significant effect on the response variables. Given the natural history of $O$. virilis, we suggest that selection has favored the ability to equally utilize chemical cues in both still and flowing water. We acknowledge that different flow conditions may influence chemical ecology in this species and caution against the view that tests in flowing waters necessarily provide a more accurate approximation of natural responses.
\end{abstract}

\section{Introduction}

The responses of prey animals to resource stimuli (e.g. food and potential mates) in the presence of predation risk depend upon the context in which those cues are encountered. If an animal encounters both a resource cue and a predation risk cue, the combination can elicit responses that fall anywhere on the spectrum between the responses elicited by these stimuli when detected alone. The internal and external environments experienced by an animal affect the decision to pursue the resource, avoid predation or exhibit an intermediate response. Investigators have considered a number of the environmental variables that can influence behavioral tradeoffs, such as starvation (Sih et al. 1991; Hazlett 2003), recent history with risk (Hamilton \& Heithaus 2001; Pecor \& Hazlett 2003), intensity of risk (Madison et al. 1999), and resource availability (Hazlett \& Rittschof 2000). In contrast, variation in the medium through which the resource and risk signals travel has received little attention. For aquatic systems, hydrodynamics may be critical to understanding tradeoffs between pursuit and antipredator behaviors.

The lack of attention paid to hydrodynamics in the context of tradeoffs is not indicative of general neglect. There is a substantial literature on the importance of flow for biological systems (e.g. Vogel 1981), and the influence of hydrodynamics on chemical signals in aquatic systems has been widely studied (see review by Moore $\&$ Crimaldi 2004). In empirical studies, flow has been shown to both improve and hinder the use of chemical cues. Oyster drills were more responsive to food cue in a high-flow environment 
than in a low-flow or still environment (Brown $\&$ Rittschof 1984). Brown bullhead catfish were better able to orient to chemical cues in still water than in flowing water, following a more direct route to a food odor source in still water (Sherman \& Moore 2001). Brown bullhead catfish inhabit still waters, so this result is consistent with the ecology of that species.

Both flowing and static experimental systems have been used to test hypotheses about the use of chemical signals in behavioral tradeoffs (e.g. Petranka et al. 1987; Hazlett 1999), but studies that compare responses between flowing and still waters are lacking. We know only two studies in which the effects of flow on chemically mediated behavioral tradeoffs were considered (Pecor 2005; Hazlett et al. in press), and flow was not isolated as the only variable in either of those studies.

We present here the results of a test of the hypothesis that the responses to either food or food plus predation risk stimuli depend upon whether these stimuli are encountered in flowing water or static water. We tested our hypothesis using the virile crayfish, Orconectes virilis. The tradeoff between pursuit of food stimuli and avoidance of predation risk stimuli, including conspecific alarm cue, has been well studied in O. virilis (e.g. Hazlett 1999). Also, this species can be found in waters that range from ponds to swiftly flowing rivers (Crocker $\&$ Barr 1968). Thus, the virile crayfish is an excellent model for a test of the influence of hydrodynamics on the tradeoff between pursuit and antipredator responses. Based on its distribution in a variety of habitats and ability to move between habitats, it could be predicted that no difference in the use of food and alarm signals would be found between habitat types. Moore \& Grills (1999) reported that an increase in turbulence increased the efficiency with which rusty crayfish (Orconectes rusticus) used chemical signals. Like $O$. virilis, the rusty crayfish can be found in a variety of aquatic habitats. Thus, we predicted that crayfish in the food treatment would be more attracted to the source of the stimulus in a flowing system than in a static system. Animals in the food plus predation risk treatment were predicted to exhibit more antipredator behavior if the cues were encountered in flowing water than if the cues were encountered in still water.

\section{Methods}

\section{Collection and Maintenance of Animals}

Crayfish were collected from the DNR Saline Fisheries Research Station in Washtenaw Co. (MI, USA - $\left.42^{\circ} 09^{\prime} \mathrm{N}, 83^{\circ} 46^{\prime} \mathrm{W}\right)$. This site was described in detail by Latta \& Merna (1977). Crayfish were housed in single-sex tanks and offered AquaMax ${ }^{\circledR}$ fish chow every few days. Individuals of $O$. virilis are motivated to forage in spite of predation risk after more than $3 \mathrm{~d}$ of starvation (Hazlett 2003), so all animals were tested within $3 \mathrm{~d}$ of their most recent feeding. Animals were collected and tested in Oct. and Nov. 2004, and these months are within the breeding season for $O$. virilis at the latitude sampled (AmeyawAkumfi 1976). In an effort to minimize variation in responses, we used only reproductive females (form I) as test subjects. Form I females were distinguished from non-reproductive females (form II) by the presence of glair glands on the telson of form I animals (Wetzel 2002). The crayfish used were of a medium size, with a $\overline{\mathrm{x}}$ carapace length of $3.7 \pm 0.02 \mathrm{~cm}$.

\section{Experimental Stream Arena}

An artificial stream arena was constructed from polyvinyl chloride (PVC) pipe, PVC valves, and vinyl tubing (Fig. 1). The arena consisted of 10 artificial

(a) Stimulus introduction apparatus

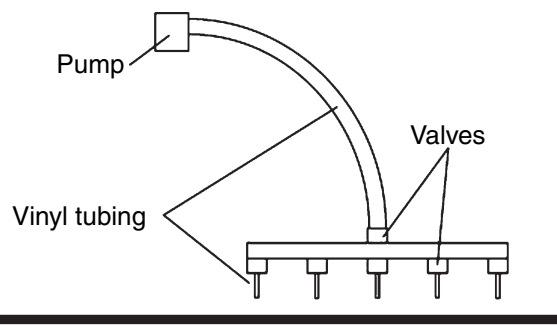

(b) Artificial stream arena

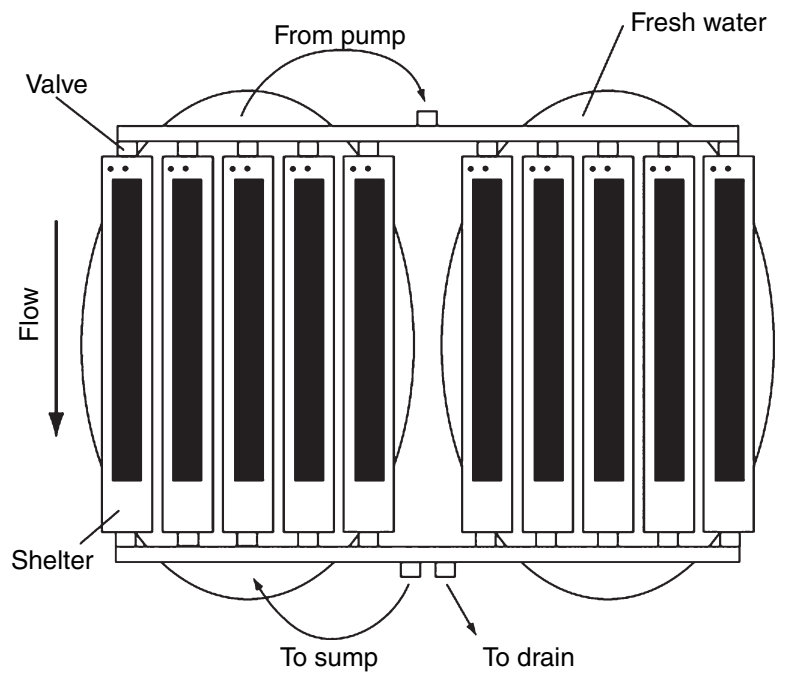

Fig. 1: The stimulus introduction apparatus (a) and artificial stream arena (b) used in this study. Neither drawing is to scale 
streams (Fig. lb) and a stimulus introduction apparatus (Fig. la). The individual streams were troughs made from PVC pipe (ID = $10.2 \mathrm{~cm})$. The streams were $1 \mathrm{~m}$ in length, and we removed a section of the pipe $7.8-\mathrm{cm}$ wide and $74-\mathrm{cm}$ long to permit overhead observation of the crayfish. Sixteen centimeters of pipe remained intact at the downstream end of the trough and provided a shelter for the crayfish. Both ends of each trough were capped with a disc of PVC, and ball valves (ID $=1.9 \mathrm{~cm}$ ) allowed for regulation of flow into and out of the troughs. The ball valves at the upstream end of the arena were fed by a PVC distribution line $(\mathrm{ID}=2.0 \mathrm{~cm})$. The ball valves at the downstream end of the arena emptied into a PVC drain line (ID = $2.7 \mathrm{~cm}$ ). Two small holes were drilled in the top of each trough at the upstream end. One of the holes accommodated airline tubing and an air stone. The other hole permitted the introduction of stimulus solutions. Stimuli were introduced via an apparatus consisting of a small-volume pump, vinyl tubing $(\mathrm{ID}=1.3 \mathrm{~cm}$ ), PVC pipe and valves (ID $=1.5 \mathrm{~cm}$ ) and airline tubing $(\mathrm{ID}=0.4 \mathrm{~cm})$ (Fig. la). The stream arena was supported by a custom-made wood table. Two $380 \mathrm{l}$ cattle tanks sat under the table supporting the arena. A third cattle tank sat adjacent to the table.

The stream operated as a recirculating system. The cattle tank pictured on the left in Fig. lb served as a sump and was filled with approx. $215 \mathrm{l}$ of water. All water was treated to remove chlorine. A continuousduty sump pump (ECOSUB 410A) was used to pump water out of the sump tank, through vinyl tubing (ID $=3.2 \mathrm{~cm}$ ), and into the distribution line at the upstream end of the arena. Water flowed through the troughs at a rate of $2 \mathrm{l} / \mathrm{min}$. This flow rate resulted in a water volume of $6 \mathrm{l}$ and a water depth of $7.5 \mathrm{~cm}$ in each trough. Water exited the troughs into the drain line and was directed into the sump.

\section{Experimental Design and Protocol}

To test for the effects of stimulus treatment and habitat type, a factorial ANOvA design was used (Zar 1999). There were two stimulus treatments, two habitat types and 20 replicates of each stimulus $\times$ habitat treatment $(\mathrm{n}=80)$. The two stimulus treatments were food cue and food + alarm cue. Food cue was generated by combining $10 \mathrm{~g}$ of ground fish chow with $1 \mathrm{l}$ of water, allowing the mixture to soak for $5 \mathrm{~min}$, and filtering the solution with cheesecloth. The filtrate was used as the cue. Alarm cue was generated by macerating a female crayfish $(\overline{\mathrm{x}}$ mass $=24.35 \pm 0.52 \mathrm{~g}, \overline{\mathrm{x}}$ carapace length $=4.2 \pm 0.07 \mathrm{~cm}$ ) in $2 \mathrm{l}$ of water, allowing the mixture to soak for $5 \mathrm{~min}$, and filtering with cheesecloth. The filtrate was used as the alarm cue.

The two habitat types were static water and flowing water. In the static treatment, the ball valves at each end of the troughs were closed completely, and each trough was filled with $5 \mathrm{l}$ of water. This volume yielded a water depth of $6.4 \mathrm{~cm}$. In the flowing treatment, the streams ran as previously described, except during testing. Two ball valves were present on the drain line (Fig. lb). During the time that crayfish were acclimating to the stream arena, the water exiting the troughs was directed into the sump. During testing, the water was directed into a drain in the floor of the laboratory. This step served two functions. First, it kept stimulus strength constant during the test by preventing stimulus accumulation in the sump. Secondly, it prevented contamination of troughs not under observation. The arena was designed so that the streams would be tested sequentially in two groups of five. If stimulus-laden water had recirculated throughout the arena, then the second group of five troughs would have been contaminated by the stimulus solution from the first group. Directing the water into a drain caused the water level in the sump to fall during testing. Each non-sump cattle tank was filled with 2151 of fresh water, and that water was pumped into the sump as needed to replenish the lost water. The fresh water was aged $23 \mathrm{~h}$ to allow it to equilibrate to the same temperature as the water in the sump.

Crayfish were given $23 \mathrm{~h}$ to acclimate to the stream arena in both the flowing and static treatments. After $23 \mathrm{~h}$, testing with stimulus solutions began. For the food treatment, the stimulus solution was $19 \mathrm{l}$ of fresh water and $1 \mathrm{l}$ of the food cue. For the food + alarm treatment, the stimulus solution was $18 \mathrm{l}$ of fresh water, $1 \mathrm{l}$ of food cue, and $1 \mathrm{l}$ of alarm cue. As mentioned above, two groups of five streams were run. Food cue was prepared fresh for each group. To minimize the number of crayfish that needed to be killed, a single alarm cue was prepared on the days that it was needed and 11 of that cue was used for each group. Alarm cue takes approx. $6 \mathrm{~h}$ to lose its effect (Hazlett 2003), and all trials were completed within approx. 20 min of alarm cue preparation. Thus, we are confident that the strength of the alarm cue did not vary substantially between the first and second groups. In the flowing trials, $20 \mathrm{l}$ of stimulus solution were made for each group of five streams. In the static trials, a single batch of 
201 was prepared, and it was then split into two batches of $10 \mathrm{l}$ each. The flowing trials needed more stimulus solution for each group of five because of the higher drip rate used in those treatments (see below).

The experimental design used the following drip rates to achieve equivalent stimulus strength in the two habitat types. In the flowing trials, stimuli were introduced into the streams at a rate of $0.4 \mathrm{l} / \mathrm{min}$, and trials were 8 -min long. Given the trough flow rate of $2 \mathrm{l} / \mathrm{min}$, the stimulus/fresh water ratio was 0.2:1 (3.2 l stimulus/16 l fresh water). The crayfish were allowed $3 \mathrm{~min}$ to acclimate to the stimulus and then observed for $5 \mathrm{~min}$. The 3 -min acclimation time also served to establish the stimulus concentration within each stream. Given the stream flow rate of $2 \mathrm{l} / \mathrm{min}$, the volume of the flowing stream (6 l) was replaced completely after $3 \mathrm{~min}$. During the $3 \mathrm{~min}$ of acclimation, the stimulus concentration was building towards a 0.2:1 ratio. At $3 \mathrm{~min}$, it reached 0.2:1, and that ratio was maintained by the incoming mixture of fresh water and stimulus. In the static treatment, the stimulus drip rate was set to $0.34 \mathrm{l} / \mathrm{min}$, and stimulus was pumped into the troughs for $3 \mathrm{~min}$. This drip rate approximated the conditions in the flowing treatment, in that the concentration was building towards $0.2: 1$ over $3 \mathrm{~min}$ and reached that ratio at $3 \mathrm{~min}$ ( $1 \mathrm{l}$ stimulus/5 1 water). Dye tests confirmed that the stimulus was able to diffuse the length of the trough in the static treatment during the span of $3 \mathrm{~min}$. After $3 \mathrm{~min}$, stimulus introduction stopped, and the crayfish were observed for 5 min.

\section{Response Variables, Observations, and Analyses}

As in other studies of crayfish chemical ecology (e.g. Pecor \& Hazlett 2003), we sought to understand the effects of our treatments on habitat use and activity. To facilitate taking these measures, each $1 \mathrm{~m}$ stream was divided into four sections of $25 \mathrm{~cm}$, and lines were drawn on the outside of the pipe to denote the sections. The section farthest upstream was termed the 'inlet' section, and the section farthest downstream was termed the 'shelter' section. The inlet section contained the hole through which the stimulus solutions were introduced, and the shelter section contained the intact pipe that served as a refuge. To assess habitat use, the time spent in the inlet and shelter sections during a 5-min observation was recorded. We predicted that the crayfish would spend more time in the inlet and less time in the shelter in the food treatment and that the opposite pattern of habitat use would be elicited by the food + alarm treatment. We predicted that the sensitivity of the crayfish to the chemical cues would be higher in the flowing trials (e.g. Moore $\&$ Grills 1999). As a result, the time spent in the inlet and shelter sections would be higher for the food and food + alarm treatments, respectively, in the flowing treatment than in the static treatment. Activity was gauged by recording the number of lines crossed during the $5 \mathrm{~min}$ of observation. A higher number of lines crossed would indicate greater movement among stream sections and hence, a higher level of activity. We predicted greater activity in the food treatment than in the food + alarm treatment. We also predicted greater activity in the static treatment than in the flowing treatment, because we thought that the information about the stimulus location present in the flow would minimize the activity needed to locate the source.

Observations were made as follows. During acclimation, the crayfish were on a 12:12-hour photoperiod, with daylight from 6:00 to 18:00 hours. The virile crayfish is nocturnal, so observations were made from 22:00 hours to midnight, aided by red lights, which crayfish cannot see. Streams were tested in groups of five, and crayfish were observed indirectly using a digital video camera with infrared capabilities (Sony DCR-TRV25). The camera was connected to a notebook computer via an IEEE 1394 cable, and trials were recorded directly to the computer's hard drive as video files using Adobe Premiere 6.5. The video files were later scored for: (1) the time spent by each crayfish in the four sections of its stream; and (2) the number of lines crossed by each crayfish. The times spent in the inlet and shelter sections of the trough were analyzed together using multivariate analysis of variance (MANOva). This test is appropriate because the measures were not independent. Time spent in one section of the stream could not be spent in the other section. Pillai's Trace was selected as the multivariate root for its robustness (Scheiner 2001) and general applicability (Zar 1999). The number of lines crossed was analyzed using a two-factor ANOVA. All statistical calculations were made using SAS v8.2.

\section{Results}

Stimulus treatment had a significant effect on the time spent in the inlet and shelter sections (Pillai's Trace $\left.=0.22, \quad F_{2,75}=10.15, \quad p<0.001\right)$. Less time was spent in the shelter section in the food treatment than in the food + alarm treatment, and time 

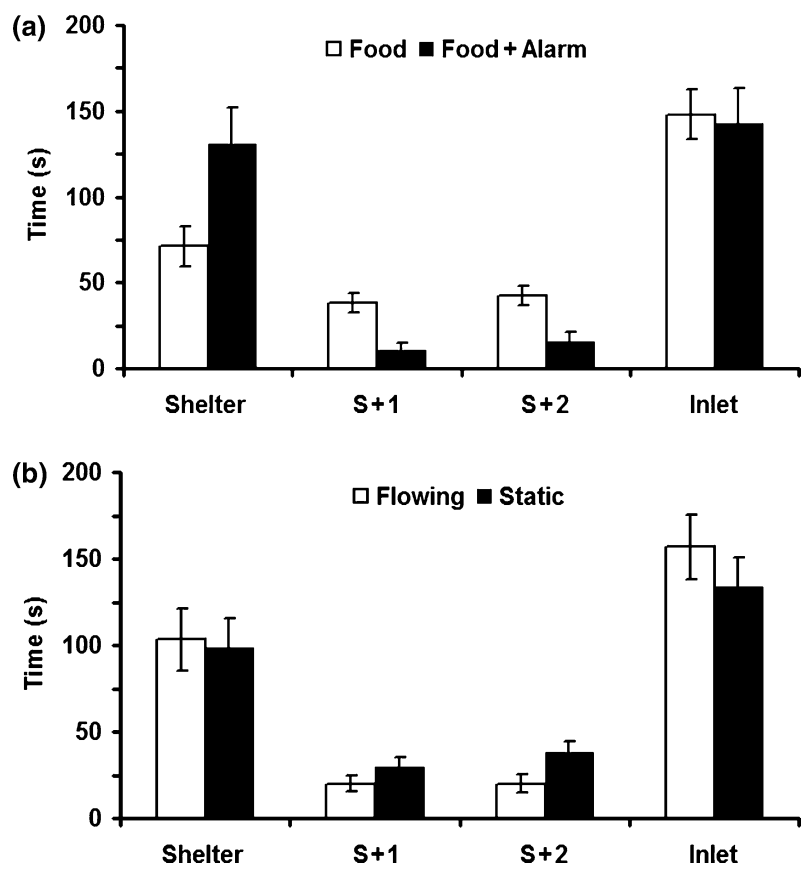

Fig. 2: The time spent in the four sections of the artificial stream between (a) the food and food + alarm stimulus treatments and (b) the flowing and static water treatments. Shelter, the section of the stream containing intact pipe; $S+1$, the first section upstream from the shelter; $S+2$, the second section upstream from the shelter; inlet, the section of the stream into which the stimuli were introduced. Plots are $\bar{x} \pm$ SE

spent in the inlet section was similar between the two treatments (Fig. 2a). Neither habitat type (Pillai's Trace $=0.06, \mathrm{~F}_{2,75}=2.35, \mathrm{p}=0.10$; Fig. $2 \mathrm{~b}$ ) nor the interaction of stimulus and habitat (Pillai's Trace $\left.=0.005, \mathrm{~F}_{2,75}=0.19, \mathrm{p}=0.83\right)$ had a significant effect on the amount of time spent in the inlet and shelter sections.

Activity was similarly affected by stimulus and habitat type. The number of lines crossed was significantly higher in the food treatment than in the food + alarm treatment $\left(\mathrm{F}_{1,76}=20.41, \mathrm{p}<0.001\right.$; Fig. 3a). Neither habitat type $\left(\mathrm{F}_{1,76}=3.42, \mathrm{p}=0.07\right.$; Fig. $3 \mathrm{~b})$ nor the interaction of stimulus and habitat type $\left(\mathrm{F}_{1,76}=1.16, \mathrm{p}=0.28\right.$; Fig. $\left.3 \mathrm{c}\right)$ had a significant effect on the number of lines crossed.

\section{Discussion}

We found that flow was not a significant influence on the responses to chemical signals by the virile crayfish. Neither habitat use nor activity was significantly affected by the habitat type in which the stimuli were encountered. In contrast, stimulus treatment had a significant effect on both habitat
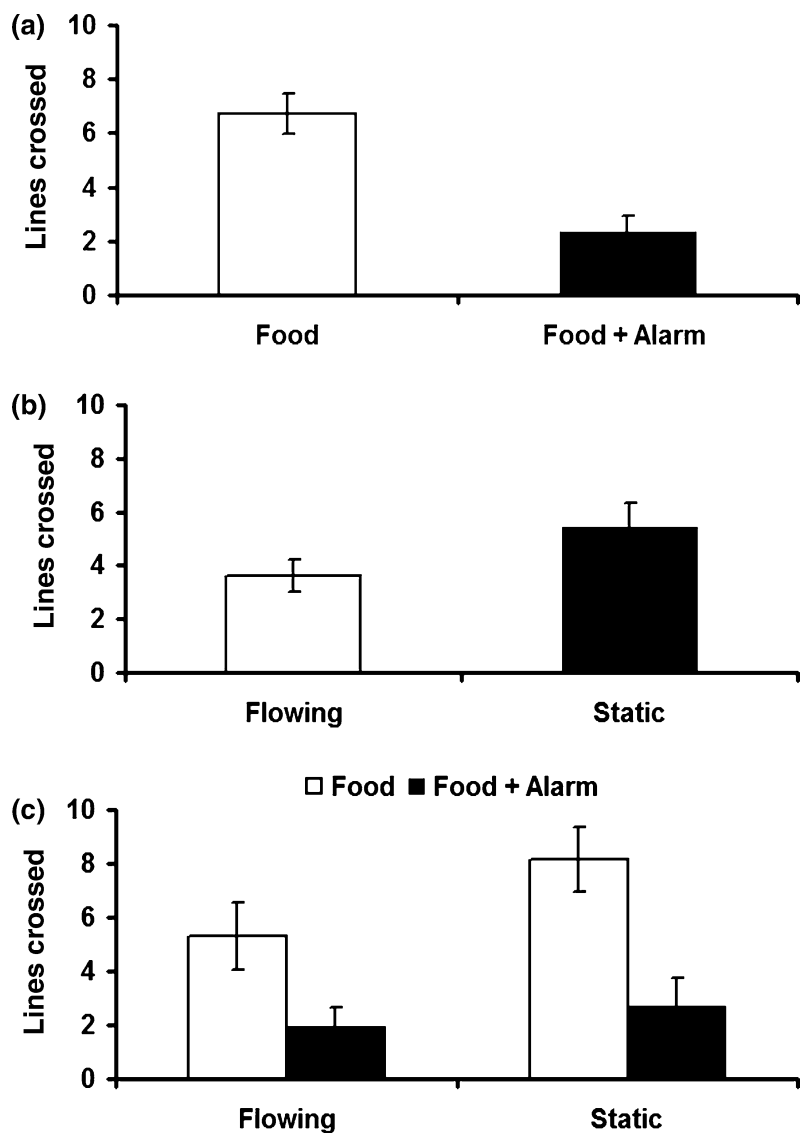

Fig. 3: The number of lines crossed between (a) the food and food + alarm stimulus treatments and (b) the flowing and static water treatments. A comparison of the responses between the food and food + alarm stimulus treatments within each habitat type is also provided (c). Plots are $\bar{x} \pm$ SE

use and activity. This latter result mirrored the results of earlier work with this species (Hazlett 1999). An unexpected finding was the lack of a qualitative difference in the time spent in the inlet section of the streams between the food and the food + alarm treatment (Fig. 2a). We suggest that this result is an artifact of the experimental arena. There was a small section of complete pipe in the inlet section that was left intact to prevent the crayfish from climbing the air stone tubing and escaping from the trough. During the food + alarm trials, many of the animals appeared to treat that area as a second shelter and did not move from that spot, as evidenced by the minimal use of the middle sections of the stream (Fig. 2a) and the low number of lines crossed in the food + alarm treatment (Fig. 3a).

It is not entirely surprising that habitat type did not have a significant effect on the responses to chemical signals, given the natural history of the 
species under study. Individuals of $O$. virilis can be found in both flowing waters (e.g. streams and rivers) and relatively static waters (e.g. ponds). We collected our study animals from farm ponds, but the Saline River borders the Fisheries Research Station on its southwest edge (Latta \& Merna 1977). The crayfish could easily travel between these sites, and the ability to use chemical signals effectively in both environments is likely to have been selected for in this species and other species with similar ecologies.

In spite of our findings, we do not rule out that flow could result in more effective use of chemical cues. We used a relatively slow flow rate in our artificial stream arena, and a faster flow could communicate more information than a slower flow. Brown $\delta$ Rittschof (1984) demonstrated that a certain threshold flow rate was needed before oyster drills responded differently to food cues in flowing water than they responded in still water. Hazlett et al. (in press) observed differences in behavior in $O$. virilis between aquarium trials and artificial stream trials. They found $O$. virilis to be more sensitive to chemical cues in the artificial streams; however, they used streams with a higher velocity than those used here. Moore et al. (1994) reported that increasing the flow rate in an artificial stream changed the structure of an odor plume, resulting in discrete 'pulses' of cue. We did not assess the structure of the odor plumes in our two treatments, but it is possible that the flow rate used in the flowing treatment did not result in a change in the signal structure when compared to the static treatment. Similarity in plume structure could explain the lack of a difference in the behaviors observed between the two treatments.

Although we acknowledge the potential for differences as flow rate increases, we suggest that flow is not always important for the end result and sometimes affects only the means to that end. We assessed an end result (i.e. the tradeoff), but did not observe the behaviors of the crayfish during the time that the stimuli were building in concentration towards the final ratio. Sherman \& Moore (2001) found differences in the ability of catfish to orient towards a food source, but they found no significant difference in the percent success of finding the source. The crayfish in our study may have differed in their ability to initially orient to the stimuli between the two habitat types (e.g. Moore \& Grills 1999), but their ultimate response to the cues was not affected by habitat type. This hypothesis deserves further testing.

We found that hydrodynamics did not influence the tradeoff between pursuit and antipredator behaviors, as measured by habitat use and activity, in the crayfish $O$. virilis. This study adds to a growing literature on the ways in which environmental variation influences behavioral tradeoffs. We advocate consideration of hydrodynamics in future studies, but we also caution against the view that results from static arenas (e.g. aquaria) are artifacts. Flow is not necessarily an important factor in all aspects of the chemical ecology of aquatic organisms.

\section{Acknowledgements}

We thank the staff of the Saline Fisheries Research Station for their help in capturing the crayfish used in this study and for permission to work at the site. P.A. Moore and J.V. Ortega helped with the calculation of drip and flow rates between habitat types. J.C. Mitani, R.A. Nussbaum, R.B. Payne, and two anonymous referees made helpful comments on earlier versions of the manuscript. This project was funded by grants from the Rackham Graduate School and Museum of Zoology of the University of Michigan to KWP.

\section{Literature Cited}

Ameyaw-Akumfi, C. E. 1976: Some aspects of breeding biology of crayfish. PhD thesis, Univ. of Michigan, Ann Arbor, MI.

Brown, B. \& Rittschof, D. 1984: Effects of flow and concentration of attractant on newly hatched oyster drills, Urosalpinx cinerea (Say). Mar. Behav. Physiol. 11, 75-93.

Crocker, D. W. \& Barr, D. W. 1968: Handbook of the Crayfishes of Ontario. Royal Ontario Museum Misc. Publ., ON, p. 158.

Hamilton, I. M. \& Heithaus, M. R. 2001: The effects of temporal variation in predation risk on anti-predator behaviour: an empirical test using marine snails. Proc. R. Soc. Lond. B 268, 2585-2588.

Hazlett, B. A. 1999: Responses to multiple chemical cues by the crayfish Orconectes virilis. Behaviour 136, $161-177$.

Hazlett, B. A. 2003: The effects of starvation on crayfish responses to alarm odor. Ethology 109, 587-592.

Hazlett, B. A., Acquistapace, P. \& Gherardi, F. in press: Responses of the crayfish Orconectes virilis to chemical cues depend upon flow conditions. J. Crust. Biol. (in press).

Hazlett, B. A. \& Rittschof, D. 2000: Predation-reproduction conflict resolution in the hermit crab, Clibanarius vittatus. Ethology 106, 811-818. 
Latta, W. C. \& Merna, J. W. 1977: Some factors influencing size of the year class of bluegills (Lepomis macrochirus) in ponds. Mich. Acad. 9, 483-502.

Madison, D. M., Maerz, J. C. \& McDarby, J. H. 1999: Optimization of predator avoidance by salamanders using chemical cues: diet and diel effects. Ethology 105, 1073-1086.

Moore, P. \& Crimaldi, J. 2004: Odor landscapes and animal behavior: tracking odor plumes in different physical worlds. J. Mar. Sys. 49, 55-64.

Moore, P. A. \& Grills, J. A. 1999: Chemical orientation to food by the crayfish Orconectes rusticus: influence of hydrodynamics. Anim. Behav. 58, 953-963.

Moore, P. A., Weissburg, M. J., Parrish, J. M., ZimmerFaust, R. K. \& Gerhardt, G. A. 1994: Spatial distribution of odors in simulated benthic boundary layer flows. J. Chem. Ecol. 20, 255-279.

Pecor, K. W. 2005: Some aspects of the chemical ecology of the crayfish Orconectes virilis and O. rusticus. PhD thesis, Univ. of Michigan, Ann Arbor, MI.

Pecor, K. W. \& Hazlett, B. A. 2003: Frequency of encounter with risk and the tradeoff between pursuit and antipredator behaviors in crayfish: a test of the risk allocation hypothesis. Ethology 109, 97-106.
Petranka, J. W., Kats, L. B. \& Sih, A. 1987: Predator-prey interactions among fish and larval amphibians: use of chemical cues to detect predatory fish. Anim. Behav. 35, 420-425.

Scheiner, S. M. 2001: MANOVA. In: Design and Analysis of Ecological Experiments, 2nd edn (Scheiner, S. M. \& Gurevitch, J., eds). Oxford Univ. Press, Oxford, pp. $99-115$.

Sherman, M. L. \& Moore, P. A. 2001: Chemical orientation of brown bullheads, Ameirus nebulosus, under different flow conditions. J. Chem. Ecol. 27, 2301-2318.

Sih, A., Krupa, J. \& Travers, S. 1991: An experimental study on the effects of predation risk and feeding regime on the mating behavior of the water strider. Am. Nat. 135, 284-290.

Vogel, S. 1981: Life in Moving Fluids. Willard Grant Press, Boston, MA.

Wetzel, J. E. 2002: Form alternation in of adult female crayfishes of the genus Orconectes (Decapoda: Cambaridae). Am. Midl. Nat. 147, 326-337.

Zar, J. H. 1999: Biostatistical Analysis, 4th edn. Prentice Hall, Upper Saddle River, NJ. 